\title{
Magnetic Suppression of Arc Blowout in a Model Arc Furnace
}

\author{
Paul M. Bellan and Jay W. Higley
}

\begin{abstract}
The two most likely causes of electromagnetic instability in electric furnace arcs are shown to be the kink and the fire-hose instabilities. Stabilization by an externally imposed axially magnetic field is analyzed, and experimental results are presented demonstrating stabilization of a small, pulsed test arc by this method.
\end{abstract}

\section{INTRODUCTION}

A TMOSPHERIC pressure high-current electric arc furnaces (EAF's) [1] are used to melt and refine scrap steel and are responsible for about one third of world steel production. Most industrial electric furnace arcs use three large (up to $0.7 \mathrm{~m}$ ) diameter graphite cylinder electrodes arranged as shown in Fig. 1. These electrodes are connected to a three-phase 50 or $60 \mathrm{~Hz}$ ac power supply so that at any instant there simultaneously exist arcs between each of the three electrodes and the melt. The arc length is continuously adjusted by mechanically raising or lowering the electrodes. Typical parameters are: arc current $\sim 3-100 \mathrm{kA}$, arc voltage drop $\sim 100-600 \mathrm{~V}$, arc length $\sim 5-20 \mathrm{~cm}$, arc radius $\sim 1-5 \mathrm{~cm}$, arc temperature $1-2 \mathrm{eV}$, arc plasma density $\sim 10^{22}-10^{23} \mathrm{~m}^{-3}$, arc power input $\sim 1-100$ MVA (with the reactive power comparable in magnitude to the resistive power). Recently, single-electrode dc arc furnaces [2] have also been built in the expectation that electrode consumption would be reduced. In contrast to the three-phase ac furnace, these dc furnaces have a cylindrically symmetric geometry.

The arc in a three-phase ac electric arc furnace is extremely unstable-it lurches about violently and uncontrollably, generating significant electrical [3] and acoustical [4] noise and often damaging the refactory material lining the interior of the furnace wall. The recent interest in dc arc furnaces has also been stimulated by the fact that the dc arc is more stable than ac arcs. This increased stability is attributed to (i) the greater symmetry of the single-electrode configuration of the dc arc (compared with the three-electrode ac arc) and (ii) the fact that the $\mathrm{dc}$ arc always has the graphite electrode as cathode (which is more stable [5] than having a steel cathode).

The physics of high current arcs has been discussed extensively by Maecker [6], Bowman, Jordan and Fitzgerald [7], Bowman [8], Jordan, Bowman, and Wakelam [9], Edels

Manuscript received February 18, 1992; revised June 16, 1992. This work was supported by NSF Grant ECS-8814184 and by a Caltech Summer Undergraduate Research Fellowship (SURF) to the second author.

P. M. Bellan is with the California Institute of Technology, Pasadena, CA 91125 .

J. W. Higley was with the California Institute of Technology, Pasadena, CA. He is now with the XonTech Corp., Los Angeles, CA 90045.

IEEE Log Number 9203923.

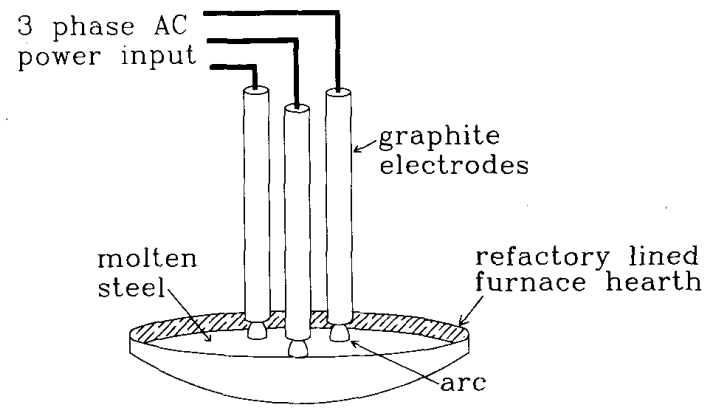

Fig. 1. Three-phase electric arc furnace geometry. Typical hearth diameter is $5-7 \mathrm{~m}$, typical hearth capacity is $50-100$ metric tons.

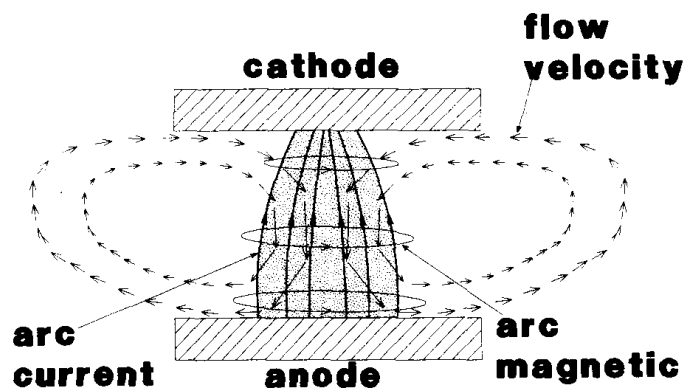

Fig. 2. Idealized geometry of arc. Conventional current (solid lines) flows from anode to cathode through arc plasma (shaded region); stronger magnetic field at cathode than at anode acts as a "pump" for fluid vortex (arrows). Fluid outside shaded region is not ionized. If the cathode starts to evaporate then an additional mass influx of evaporated material (electrode jet) will also participate in the flow.

[10], Irie and Barrault [11], Strachan [5], McKelliget and Szekely [12], Jones [13], and Ochs [14]. Maecker identified the dominant feature of the high current arc-an acceleration of plasma from cathode to anode due to the gradient of the arc self magnetic field from cathode to anode. If the current becomes constricted (pinched) at the anode, similar anode jets can form having flow away from the anode. In fact [6], the acceleration mechanism is solely due to current being constricted, and the acceleration direction is always away from the constriction, regardless of the polarity of the current. Very high fluid velocities (several $\mathrm{km} / \mathrm{s}$ ) result [8] from this acceleration, and in a high-current arc most [10] of the electrical energy input goes into translational kinetic energy rather than into thermal energy or radiation. For graphite cathodes the actual accelerated fluid is plasma produced from neutral gas streaming into the cathode region, 


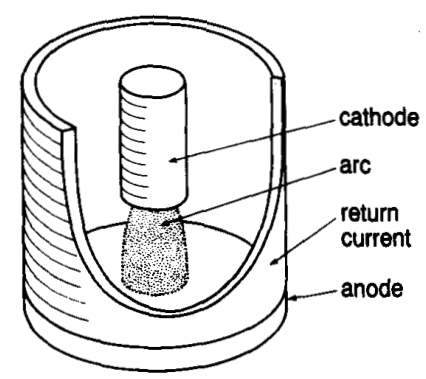

(a)

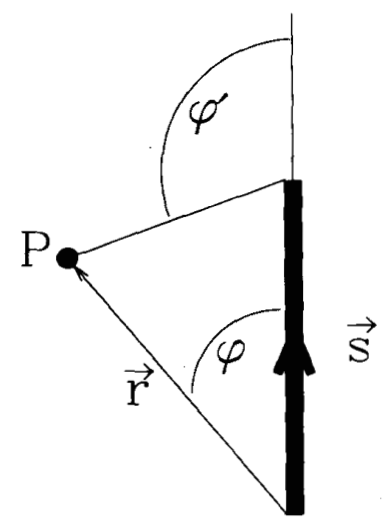

(c)

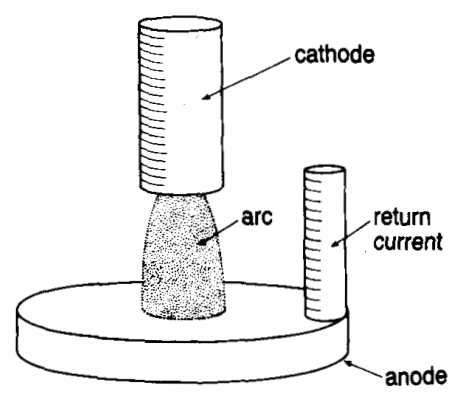

(b)

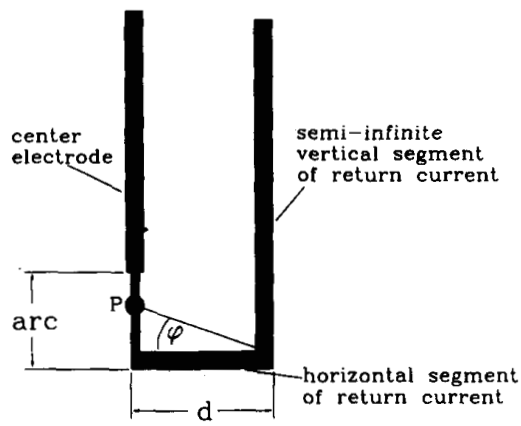

(d)

Fig. 3. (a) Symmetric return current; (b) asymmetric return current; (c) current segment $\vec{s}$ and observation point $P$ for Biot-Savart law, Eq. (1); (d) idealized asymmetric arc geometry for Biot-Savart calculation leading to Eq. (2).

and not (as might be intuitively expected) material ablated from the cathode [9]. However, for metallic electrodes with very high currents, significant localized evaporation typically occurs resulting in a core plasma jet of metallic electrode material which is accelerated away from the electrode in addition to the entrained neutral gas [13].

Thus, there is a flow [12] as shown in Fig. 2, neutral gas streaming toward the cathode is ionized to form plasma, the plasma is accelerated from cathode to anode, the high-velocity plasma jet impinges on the anode where its kinetic energy is turned into heat, and a low-velocity plasma jet moves radially out from the point of impingment, becomes deionized, and ultimately flows back to the cathode. The flow has the shape of a vortex, and the magnetic force acts as a pump to sustain the vortex.

\section{MECHANISM OF THE MAGNETIC INSTABILITY}

We discuss in this section the causes of electromagnetic instability of the arc in the electric furnace. Note that previous discussions [16], [17] of arc MHD instabilities have been restricted to much lower current arcs, typically operating at low pressure, and have been essentially phenomenological. Also note that there may be nonelectromagnetic forms of instability, e.g., those associated with slag and electrode evaporation effects, and those associated with geometric irregularities in the furnace load before complete melting has taken place.

We first draw attention to the geometry of the return current since this has an important influence on arc magnetic stability. Parts (a) and (b) of Fig. 3 show the two limiting forms of return current geometries, namely perfectly symmetric and maximally asymmetric. The magnetic force, $\vec{F}$, acting on a current density, $\vec{J}$, arising from an external magnetic field, $\vec{B}$, is $\vec{F}=\vec{J} \times \vec{B}$. The force direction is such that parallel currents attract, whereas opposing currents repel. Thus, the force between the arc and its return current is always repulsive. This magnetic force can equivalently be expressed in tensor notation as $\vec{F}=-\nabla \cdot\left(B^{2} \stackrel{\leftrightarrow}{I} / 2-\vec{B} \vec{B}\right) / \mu_{0}$, where the quantity $B^{2} \overleftrightarrow{I} / 2-\vec{B} \vec{B}$ is the magnetic stress tensor. Examination of the magnetic stress tensor shows that the magnetic field exerts a pressure $B^{2} / 2 \mu_{0}$ in the direction perpendicular to $\vec{B}$ and a tension of $B^{2} / 2 \mu_{0}$ in the direction parallel to $\vec{B}$, resulting in the well-known analogy that magnetic field lines behave like rubber bands. Using the stress tensor point of view, the force between the arc and its return current is seen as a magnetic pressure localized between the two currents which pushes the two currents apart.

In Fig. 3(a) the return current of the arc is azimuthally symmetric; this makes the magnetic field arising from the return current vanish inside the return current cylinder so that 
the return current exerts no force on the arc. On the other hand, in Fig. 3(b), the return current does exert a net force on the arc. The magnitude of this force can be calculated using the Biot-Savart law, which gives the magnetic field $\Delta \vec{B}$ at a point $P$ caused by a line segment of current $\vec{s}$ as

$$
\Delta \vec{B}=\frac{\mu_{0} I\left(\cos \phi-\cos \phi^{\prime}\right)}{4 \pi r \sin \phi} \frac{\vec{s} \times \vec{r}}{|\vec{s} \times \vec{r}|},
$$

where $I$ is the current in the line segment, and the angles and $\vec{r}$ are shown in Fig. 3(c). If the arc deviates from being coaxial with the feed current (left line above arc in Fig. $3(d))$ by an angle $\delta$, then [18] (1) shows that the field at a point on the arc arising from the feed current is given by $B=\mu_{0} I(1-\cos \delta) / 4 \pi r$, where $r$ is the distance to the bottom point of the feed current. The direction of this field is such as to provide an aligning force on the arc, strongest at the top of the arc, and tending to make the arc coaxial with the feed current in the center electrode. Thus, the arc feed current does not exert a force on the arc when the arc is coaxial (i.e., $\delta=0$ ) with the feed current.

The return current consists of two line segments as shown in Fig. 3(d), a semi-infinitely long vertical segment and a short horizontal segment. Using (1) at the point $P$ on the arc, it is seen that the vertical return current segment produces a magnetic field $\Delta B=\mu_{0} I(1+\sin \phi) / 4 \pi d$, where $\phi$ is indicated in Fig. 3(d) and $d$ is the horizontal separation between the arc and the vertical return current segment. Similarly it is seen that the short horizontal segment produces at $P$ the magnetic field $\Delta B=\mu_{0} I \cos ^{2} \phi / 4 \pi d \sin \phi$. Thus the total field at $P$ is

$$
B=\frac{\mu_{0} I(1+\sin \phi)}{4 \pi d \sin \phi} .
$$

Because of the inverse $\sin \phi$ dependence we see that this field is much stronger at the bottom of the arc than at the top. The divergence of $1 / \sin \phi$ at $\phi=0$ results from the fact that there is zero spacing between the left-hand end of the horizontal segment ad the bottom of the arc; hence, at the bottom of the arc the magnetic field caused by the horizontal return current segment is infinite. In reality, the field is not infinite because the horizontal return current actually flows through the entire plane of the anode and is not a line segment. Nevertheless, the divergence resulting from the simple line-segment model alerts us to the fact that the destabilizing magnetic field acting on the arc bottom will be much larger than at the top because the bottom is in contact with the horizontal return current, whereas the top is some distance away. In contrast, the stabilizing aligning force caused by the current in the feed will be stronger at the top of the arc than at the bottom.

The current density in the arc is just $J=I / \pi a^{2}$, where $a$ is the arc radius. Hence, the force density exerted on the arc is $F=\mu_{0} I^{2}(1+\sin \phi) / 4 \pi^{2} a^{2} d \sin \phi$. If the arc mass density is $\rho$, then the arc will be accelerated away from the return current at the rate $d U / d t=\mu_{0} I^{2}(1+\sin \phi) / 4 \pi^{2} a^{2} \rho d \sin \phi$.

Because of the $(1+\sin \phi) / \sin \phi$ factor the acceleration of the arc is nonuniform along its length; i.e., the bottom of the arc accelerates much faster than the top (because of the effect of the closer proximity of the horizontal return current to the arc bottom than to the arc top, and also because the

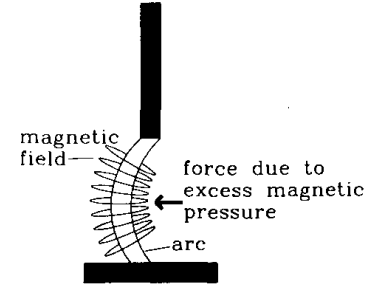

(a)

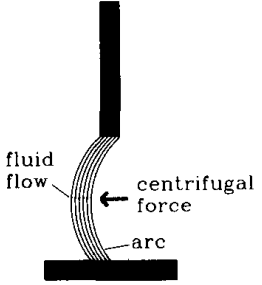

(b)
Fig. 4. (a) Kink instability-excess magnetic pressure on the inside of a curved current channel pushes the channel to increase the bending. (b) Fire-hose instability - centrifugal force due to fluid motion along the current channel similarly pushes the channel to increase the bending.

aligning force due to the feed current is stronger at the top than at the bottom). The result is that the arc becomes curved; this curved arc is strongly unstable with respect to the kink [19] and the fire-hose [20], [21] instabilities, both of which are very fast. The kink instability (cf. Fig. 4(a)) is caused by a buildup of magnetic pressure on the inside of a curved current channel; this enhanced magnetic pressure pushes the current in a direction to increase the bending, resulting in a fast growing instability. The fire-hose instability (cf. Fig. 4(b)) is similar except that centrifugal force arising from fluid flow along the curved arc does the pushing instead of magnetic pressure imbalance. Because these instabilities involve the arc interacting with itself rather than with the more distant return current, the forces driving the instabilities exceed the force due to interaction with the return current. Thus, the role of the return current is to produce the initial curvature of the arc; this provides the appropriate starting condition for the faster growing kink or fire-hose instabilities. If the return current is symmetric, kink and fire-hose instabilities may still occur, but now the initial curvature must be instigated by random noise. Contemporary dc arc furnaces do not have perfectly symmetrical return currents; however the return current is much farther away from the arc than for the three-phase ac arc (outside the furnace rather than inside), so that the instigating push is smaller.

The arc always starts at the location where the anode and cathode are closest together because here the electric field is largest, and so breakdown is easiest. When the arc bows out and becomes unstable it lengthens, increasing its resistance, thus decreasing the current. The rapid current reduction causes a sharp $L d I / d t$ voltage spike which has polarity such as to sustain the arc. Typically the arc bows out so drastically that it "blows out" to the side and extinguishes. If the voltage spike associated with the arc extinguishing is large enough, breakdown is reinitiated where the cathode and anode are closest together, forming a new arc. Hence, the death of one arc blowing out to the side contains the regeneration mechanism for a new arc located where the cathode and anode are closest together. A "continuous" arc often really consists of a continuous sequence of arcs, forming, blowing out to the side, extinguishing, and being replaced by new arcs. Large voltage spikes occur repeatedly in this situation.

If we consider the arc from one electrode in a three-phase furnace arc, we see that the return current is carried by the 
arcs from the other two electrodes. Hence, all three arcs repel each other. Furthermore, as they bend due to their mutual repulsion, they all become susceptible to the kink and fire-hose instabilities.

Two methods have been previously used to stabilize medium- and high-current arcs, namely wall stabilization and gas injection [16] stabilization. Wall stabilization involves surrounding the arc with a close fitting flux conserving wall so that image currents in the wall provide a restoring force which stabilizes the kink (and presumably fire-hose) instabilities. Wall stabilization is clearly unsuitable for the high-current furnace arc because of the impossibility of surrounding the arc with a close-fitting material wall. Gas injection involves surrounding the arc with a concentric jet of high-velocity neutral gas; the flow rates are prohibitively large for a large furnace arc, and also placing gas nozzle injectors in the arc vicinity is difficult. In the next two sections we present a third method, magnetic stabilization [22], which we believe offers the possibility for practical stabilization in an actual furnace environment.

\section{STABILIZATION OF ARCS VIA AN EXTERNALly APPLiEd Magnetic FIELD}

The kink instability [19] has been thoroughly studied both experimentally and theoretically in the context of magnetic fusion research; in fact the largest magnetic field of tokamaks has the purpose of stabilizing kinks. The fire-hose instability [20], [21] has also been studied in the fusion context for the special situation where the thermal pressure parallel to the magnetic field greatly exceeds the thermal pressure perpendicular to the magnetic field. The situation in the furnace arc is different in that the thermal pressure is isotropic, but there is now a very high velocity fluid flow, which performs a role similar to pressure anisotropy.

For both kink and fire-hose instabilities, an externally imposed magnetic field aligned parallel to the direction of current flow produces a strong stabilizing influence. This stabilization results from the fact that, as noted earlier, the magnetic field has a tension in the direction along the field. Thus, an externally imposed magnetic field, coaxial with the arc current, acts like a stretched rubber band immersed in the arc. Because the arc is a good conductor, it is a magnetic flux conserver, and so is "frozen" to the magnetic field in which it is immersed. When the arc starts to bend as it becomes unstable, it stretches the externally applied magnetic field (like stretching the rubber band), resulting in a restoring force that pushes the arc back to its initial position.

We now introduce a cylindrical coordinate system $r, \theta, z$ with the $z$ axis aligned along the arc; hence the arc self-field is $B_{\theta}$, while the externally applied field is $B_{z}$. Detailed analysis [19] shows that to stabilize the kink instability, one must have

$$
B_{z}>\left[\frac{h}{\pi r}\right] \frac{\mu_{0} I}{2 \pi r}
$$

where $h$ is the arc length, $r$ is the arc radius, and $I$ is the arc current (this equation is the Kruskal-Shafranov kink stability criterion). We present in Section $\mathrm{V}$ a detailed derivation of the

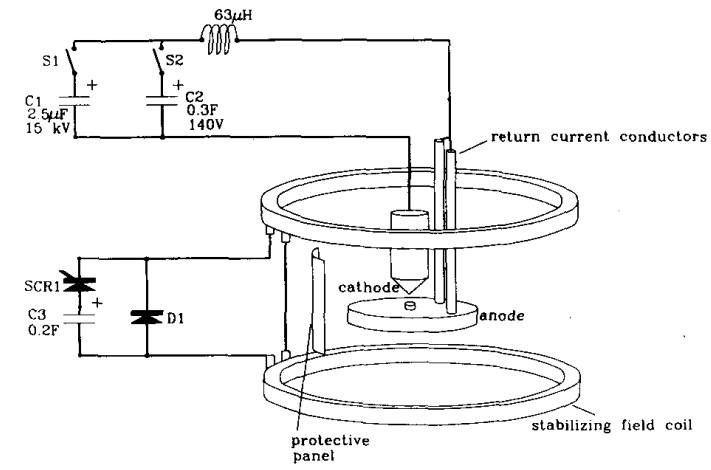

Fig. 5. Experimental setup showing arc electrodes, stabilizing field coils, and circuitry. Anode ( $77 \mathrm{~mm}$ diameter) is made of 6 -mm-thick molybdenum except for the central button ( $4 \mathrm{~mm}$ high, $1 \mathrm{~cm}$ diam), which is tungsten-copper. The $3-\mathrm{cm}$-diameter cathode is graphite and has a conical shape near the anode. Both anode and cathode have grooves (not shown here) from their center going to left to make the trajectory of the unstable arc more reproducible. The arc return current flows in two 6-mm-diameter copper rods, each $32 \mathrm{~mm}$ from the arc, and forming a right angle triangle with the arc at the right angle.

fire-hose stability criterion relevant to the arc; this derivation shows that to stabilize against the fire-hose instability one must have

$$
B_{z}>\left[\frac{h}{r}\right]^{1 / 2} \frac{\mu_{0} I}{2 \pi r} .
$$

Since $h / r \sim 1-3$ typically, we see that for both kink and fire hose the stabilizing field has to be of the same order as the arc's internal field, $B_{\text {arc }}=\mu_{0} I / 2 \pi r$. It must be realized that (3) and (4) have been derived on the basis of highly idealized assumptions, and so should be understood to give order of magnitude accuracy only.

\section{EXPERIMENTAL Demonstration of ARC STABILIZATION USING EXTERnal AXIAL MAGNETIC FIELD}

To investigate arc stability experimentally, we have constructed a small pulsed arc system with additional coils to generate the stabilizing axial magnetic field; the arc, stabilizing coils, and power supply circuitry are shown in Fig. 5. The anode-cathode separation is $6 \mathrm{~mm}$, peak arc power is $~$ $200-300 \mathrm{~kW}$, arc duration is $5-40 \mathrm{~ms}$, arc currents are $\sim 1-2$ $\mathrm{kA}$, and arc voltages are $\sim 50-100 \mathrm{~V}$. The arc is initiated by closing ignitron switch $\mathrm{S} 1$, which discharges capacitor $\mathrm{C} 1$ across the arc gap. Small capacitor $\mathrm{C} 1$ is typically charged to $\sim 15 \mathrm{kV}$, which is more than adequate to break down the air in the gap and initiate the arc. In the $\sim 20 \mu$ s quarter cycle time of the resonant circuit the arc current rises to its maximum value while the voltage on $\mathrm{C} 1$ rings down toward zero. When the voltage on $\mathrm{C} 1$ drops to $\sim 100 \mathrm{~V}$, ignitron switch $\mathrm{S} 2$ is closed, connecting large capacitor $\mathrm{C} 2$ to sustain the arc for $5-40 \mathrm{~ms}$. The return current (flowing in two rods to the right of the arc, cf. Fig. 5) has purposely been made asymmetrical to model the return current geometry of a three-phase ac arc furnace. The stabilizing coils are powered by capacitor $\mathrm{C} 3$ (charged to 0-100 V), which is switched by silicon controlled rectifier SCR1; the coil current is prevented from ringing by 


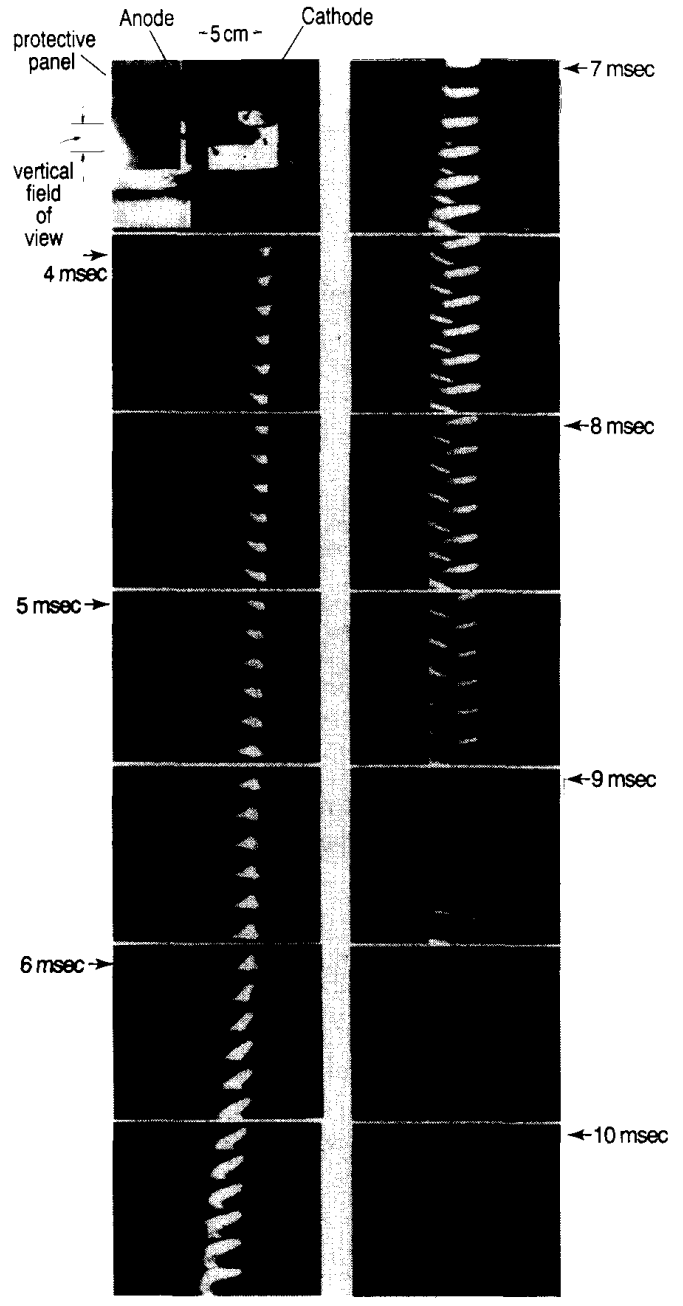

Fig. 6. High-speed video camera photos of unstable arc $(83 \mu$ s between frames). The top left shows electrode structure; arc photos have a vertical field of view that is one sixth of this photo, as indicated. Time is measured relative to the start of stabilizing magnetic field (cf. Fig. 8); are is fired at $t=4 \mathrm{~ms}$.

crowbar diode D1. The stabilizing coil circuit is triggered 4 ms before the arc is triggered to allow time for the stabilizing coil current to build up.

Measurements were made with various charging voltages on C3 to give various values of stabilizing magnetic field. Photographs of arc motion and instability were made with (i) a low-resolution $(4 \times 8$ pixel), but high-speed (up to 40000 frames/second) all digital CAMAC based framing camera, and (ii) a medium-resolution ( 32 pixel $\times 200$ pixel), high-speed (12000 frames/second) video camera. In addition, the arc current and arc voltage were recorded on transient digitizers associated with the low-resolution camera. The photographs and the digitizer signals all show that without stabilization, the arc initially moves away from the return current, then bends violently on a very fast time scale, and finally blows out to the side (left side here, since return current is on the right). This

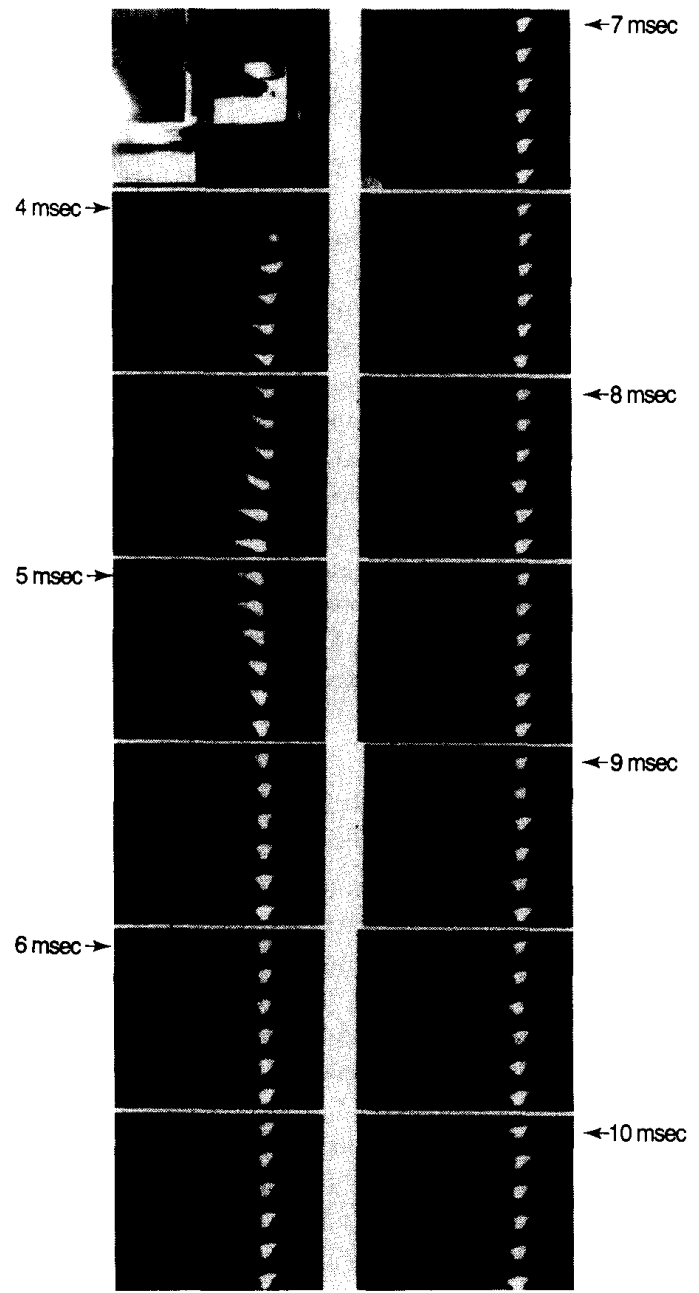

Fig. 7. High speed video photographs of stabilized arc (compare to unstabilized arc shown in Fig. 6).

instability occurs on most shots; however, there are occasional shots where the arc moves slightly to the left and then stays there without becoming unstable. The arc often extinguishes when blowing out to the left, resulting in a voltage spike and creation of a new arc at the location where the anode and the cathode are closest together (button). However, above a certain threshold charging voltage on $\mathrm{C} 3$, the arc remains relatively stable. Details of these measurements are discussed in the next two paragraphs.

Fig. 6 shows photos of an unstabilized arc (no charge on C3) measured by the high-speed video camera. The top left photo in Fig. 6 shows the electrode structure and also the vertical field of view for the arc photos. The arc first forms between the closest points of the cathode and anode, moves to the left, and then bends violently. It bends so drastically that it bounces off an insulated protective panel inserted to prevent the arc from striking the magnet coil support structure (the panel is shown in Fig. 5 and also appears as a thick white vertical line in the electrode layout photo in Fig. 6). After bouncing from the 
(a)

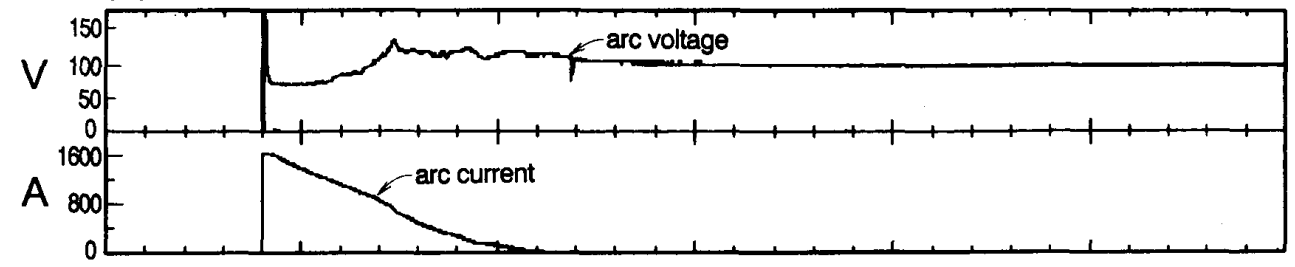

(b)

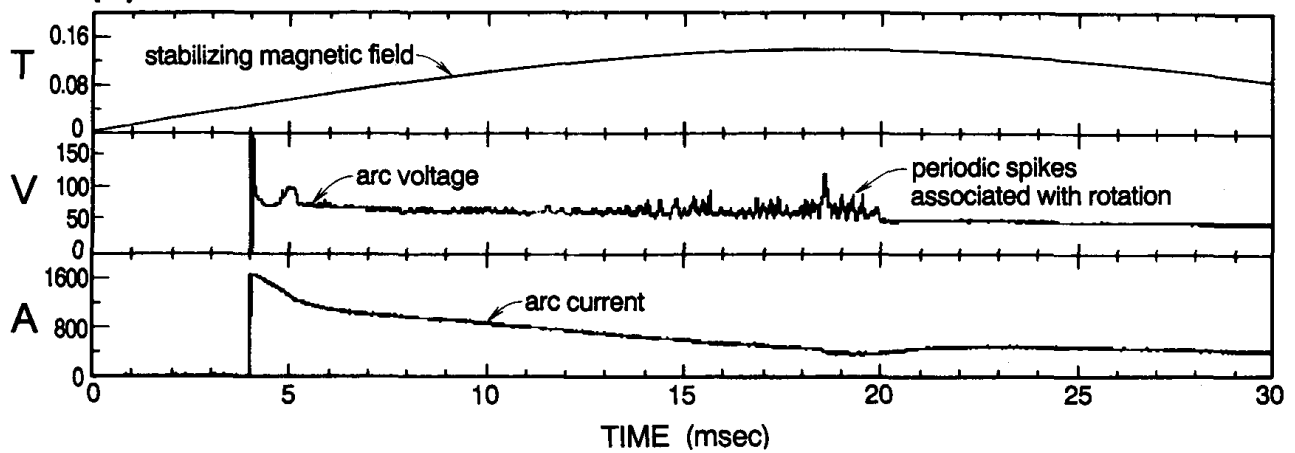

Fig. 8. (a) Voltage and current waveforms of arc in Fig. 5. (b) Voltage, current, and stabilizing magnetic field waveforms of arc in Fig. 6.

protective panel the arc strikes the anode. As expected for an instability, the exact details of the arc motion are not precisely reproducible from shot to shot. The violent bending to the left shown here is typical; variations in behavior include the arc extinguishing after it hits the protective panel, and then restriking at the location where the cathode and anode are closest together (sometimes going through several cycles of bending, extinguishing, and restriking).

Fig. 7 shows photographs of a stabilized arc (C3 charged so that peak stabilizing field is $0.1 \mathrm{~T}$ ). It is clear that application of the external magnetic field has profoundly changed the character of the arc. It no longer blows violently to the side as it did before (although it initially bends slightly to the side), and it generally stays located where it initially formed, namely the place where the cathode and anode are closest together. The arc is more compact, and generally lasts longer than the unstabilized case. It is also observed that when the arc finally extinguishes, the remaining voltage on capacitor $\mathrm{C} 2$ is lower, indicating that the arc has drawn more charge from the capacitor.

Voltage and current waveforms for the arcs shown in Figs. 6 and 7 are presented in, respectively, parts (a) and (b) of Fig. 8. Fig. 8(b) also shows the time dependence of the stabilizing magnetic field.

Late in the discharge and at relatively high external magnetic fields, a new behavior is observed, namely a helical deformation of the arc rotating at high frequency (several $\mathrm{kHz}$ ) while the arc mean position stays localized at the place where anode and cathode are closest together. This rotation is related to the periodic voltage spikes indicated in Fig. 8(b). Photographs of the rotation are shown in Fig. 9 (video camera) and Fig. 10 (CAMAC camera). Fig. 9 also shows the arc voltage and current; from these measurements it is seen that the voltage spikes are synchronous with the rotation, but unlike the voltage spikes associated with an arc extinguishing, here the arc current remains constant. Similar periodic voltage spikes and helical rotations have been seen in low pressure arcs with symmetric return currents and axial magnetic fields by Sawyer, Scott and Stratton [23] and by Yoshikawa and Irie [24]. Studies of rotary arc circuit breakers (which have coaxial geometry and axial magnetic fields) by Spencer, Parry, and Jones [26] and by Jones [27] also show helical arc rotations in axial magnetic fields. Rotating arcs have also been seen by Montgomery and Sharp [18] when there is no axial field (in [18] no mention is made of the return current geometry and the photographs suggest that the rotation is a variation of the kink instability). Jones, Eng, and Leclerc [28] postulated there might be a swirling motion in gas blast circuit breakers, while Jones, Shiskin, and Taylor [29] presented measurements of voltage oscillations in these breakers. Gas blast circuit breakers however, do not have an axial magnetic field and are wall stabilized (close fitting copper annulus around the waist of the arc) so it is likely that the mechanisms involved in gas blast circuit breakers are different. A discussion of the likely mechanism of the helical rotation in our experiment is given in Section VI.

The threshold magnetic field for stabilization can be estimated either by looking for marginal stability in a single shot or by looking at the transition from unstable to stable shots. Let us consider both methods:

Marginal Stability in a Shot: From Fig. 7 and Fig. 8(b), it is seen that about $1 \mathrm{~ms}$ after the arc starts (i.e., frames $10-12$ in Fig. 7 and at $\sim 5 \mathrm{~ms}$ in Fig. 8(b)), there is a minor instability of the arc consisting of the arc blowing slightly 


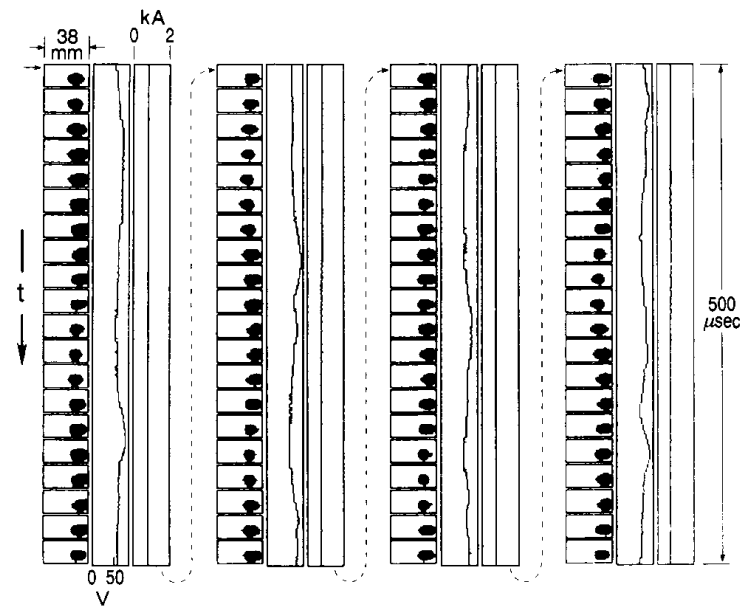

Fig. 9. Low-resolution photograph of rotating arc in high magnetic field (25 $\mu$ s between frames). Each frame shows a $19 \mathrm{~mm}$ high by $38 \mathrm{~mm}$ wide view of the arc; the cathode location is in the top right, while the anode plane is at the bottom. Rotation is manifested by left to right wobble of black blob (arc). Also shown are arc voltage and current waveforms.

to the left in the photo and a slight voltage spike. Using arc height $h \approx 6 \mathrm{~mm}$, arc radius $a \approx 3 \mathrm{~mm}, I=1.2 \mathrm{kA}$ in (3) gives the magnetic field required for kink stability to be $0.05 \mathrm{~T}$. From the top waveform in Fig. 8(b) it is seen that the actual magnetic field was indeed $0.05 \mathrm{~T}$, indicating close agreement.

Transition from Unstable to Stable Shots: Here the threshold magnetic field required for stabilization is determined using computer analysis of data from the low-resolution camera (four pixel vertical by eight pixel horizontal array). The photodetector signals at a particular horizontal position are computer averaged both vertically and over the entire arc duration for a sequence of arc shots having the same charging voltages on $\mathrm{C} 1$ and $\mathrm{C} 2$ but differing charging voltages on C3. Fig. 11 plots bar charts of these averages; here the bar charts have been offset vertically in proportion to the peak magnetic field produced by the stabilizing coils, and for clarity, only bars with amplitude exceeding $30 \%$ of the maximum amplitude of their respective shot are plotted. From Fig. 11 it is seen that for peak fields less than $\sim 0.1 \mathrm{~T}$, the arc is unstable (bar charts are to left and spread out), whereas above $\sim 0.1 \mathrm{~T}$, the arc is stable (arc stays to right, and is sharply defined). Since the magnetic field at the time the arc starts is about half the peak field (cf. top waveform in Fig. 8(b)) and since the arc current monotonically decreases with time, the arc is most vulnerable to instability during the first few milliseconds (this is simply a consequence of the waveforms here and not a general property). Again using in (3) an arc radius of $r \approx 3 \mathrm{~mm}$ and an arc height of $6 \mathrm{~mm}$ (anode-cathode separation at button), $I \approx 1.2 \mathrm{kA}$ (arc current during first millisecond of arc) gives a predicted kink stabilizing field of $0.05 \mathrm{~T}$ (recall that the stabilization condition for the fire hose is similar). This agreement between experiment and theory is considered good considering the idealized nature of the theory and the uncertainties in the arc radius and length.

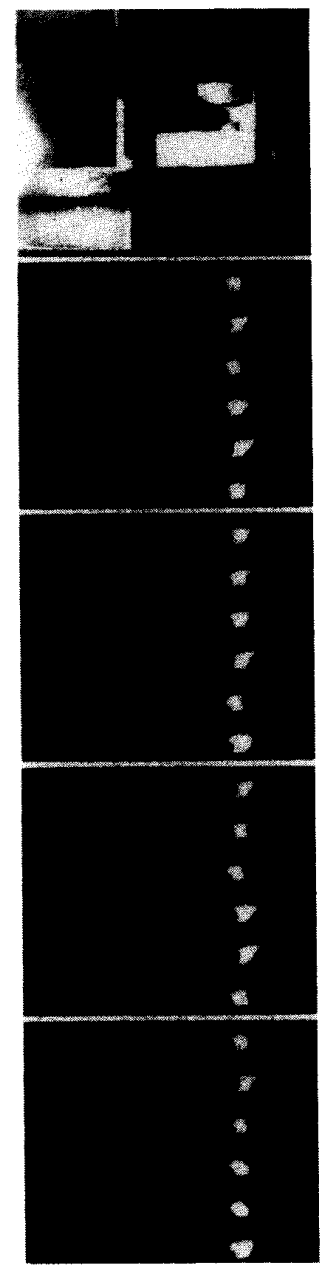

Fig. 10. High-speed video photographs of rotating arc $(83 \mu \mathrm{s}$ between individual arc images)

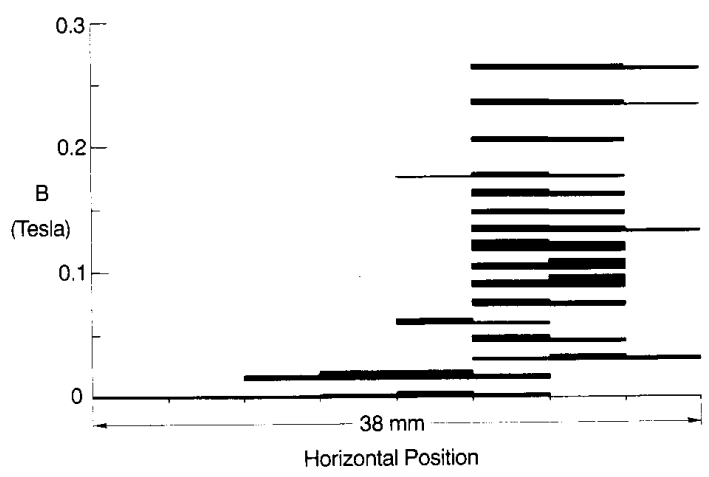

Fig. 11. Bar chart of vertically averaged, time-averaged light signal at the eight horizontal pixel locations of the low-resolution camera. For clarity and to highlight arc location, only bars with amplitude exceeding $30 \%$ of maximum amplitude for the respective shot are plotted. 


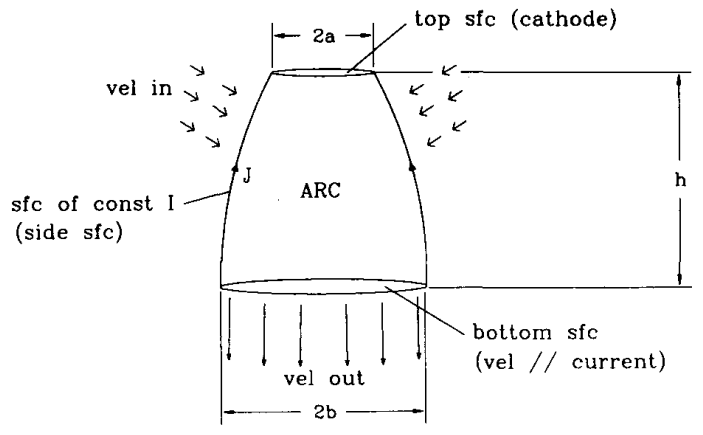

Fig. 12. Volume of integration for (5).

\section{DeRIVATION OF the Fire-Hose InStability}

A fundamental feature of these arcs is the magnetic acceleration of plasma at the cathode to very high velocities; in fact, the kinetic energy of this high velocity fluid accounts for most [10] of the arc energy content.

To calculate the fluid velocity we will make the following simplifying assumptions:

i) forces due to thermal pressure can be neglected compared to magnetic forces;

ii) the arc current density, $J$, depends only on $z$, the distance from the anode; i.e., if $r$ is the outer radius of the arc at a particular $z$, then $J=I / \pi r^{2}$. Thus, the magnetic field at a point $r^{\prime}$ inside the arc is $B=$ $\mu_{0} I r^{\prime} / 2 \pi r^{2}$.

For steady state the MHD equation of motion $\partial(\rho \vec{U}) / \partial t+$ $\nabla \cdot(\rho \vec{U} \vec{U})=\vec{J} \times \vec{B}$ becomes

$$
\nabla \cdot(\rho \vec{U} \vec{U})=-\frac{1}{\mu_{0}} \nabla \cdot\left[\overleftrightarrow{I} \frac{B^{2}}{2}-\vec{B} \vec{B}\right] .
$$

We integrate (5) over the arc volume shown in Fig. 12; this volume is bounded from the top by the cathode of radius $a$, on the side by a surface of constant current $I$, and from the bottom by a surface of radius $b$ parallel to the anode surface but located at the point where the streamlines of fluid motion are parallel to the current vector on the side (this point is roughly at the midpoint of the arc). The location of this bottom surface has been chosen so that all high-speed fluid flux exits through it, while all low-speed fluid flux enters the side surface above it. The vertical separation between top and bottom surfaces is $h$, which is about half the total arc height, and typically $h \gg b-a$.

Using Gauss's theorem over this volume of integration gives

$$
\int d \vec{S} \cdot \rho \vec{U} \vec{U}=-\frac{1}{\mu_{0}} \int d S \frac{B^{2}}{2}
$$

where the surface integrations are over the surface, side, and bottom surfaces. On the other hand, mass conservation obtained by integrating $\nabla \cdot(\rho \vec{U})=0$ over the same volume gives $\int_{\text {side }} d \vec{S} \cdot \rho \vec{U}=-\int_{\text {bot }} d \vec{S} \cdot \rho \vec{U}$. Since the surface integrals in (6) have an extra factor of $U$, and since $U$ is much larger at the bottom surface, we see that we can neglect the side surface integral in (6) compared with the bottom surface

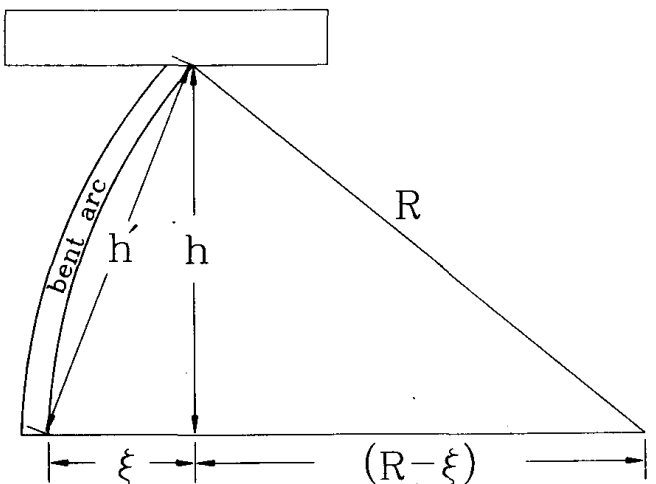

Fig. 13. Bent arc, showing how radius of curvature relates to height, displacement, and stretching. The variation in arc radius from cathode to anode has been omitted for clarity.

integral. Consequently, the only nonzero surface integrals in (6) are the bottom surface (LHS of equation only) and the side surface (RHS of equation only). Evaluating these two terms and assuming that the velocity is uniform over the bottom surface gives

$$
\begin{gathered}
\rho U^{2}=\left[\frac{\mu_{0} I}{2 \pi b}\right]^{2} \frac{h}{(b-a)} \frac{\ln (b / a)}{\mu_{0}} \\
\approx \frac{1}{\mu_{0}}\left[\frac{\mu_{0} I}{2 \pi b}\right]^{2} \frac{h}{a} .
\end{gathered}
$$

In evaluating the side surface integral we have used $d S \approx$ $2 \pi r h(b-a)^{-1} d r$; i.e., the outermost constant $I$ surface has been approximated as a straight line.

Thus, the downward fluid velocity $U$ at the bottom surface is

$$
U=V_{A}(h / a)^{1 / 2}
$$

where $V_{A}=\mu_{0} I / 2 \pi b \mu_{0}^{1 / 2} \rho^{1 / 2}$ is the Alfvén velocity calculated with respect to arc parameters at the bottom surface and $I$ is the arc current. Since $h / a \sim 2-3$ typically, we see that the arc velocity will be of the order of $V_{A}$.

Let us now assume that the arc is immersed in an externally generated axial magnetic field, $B_{\text {ext }}$, which, because of the arc's high conductivity, is "frozen" into the arc. Recall that this field has an axial tension $B_{\text {ext }}^{2} / 2 \mu_{0}$. Consider a slight bending of the arc as shown in Fig. 13; here the radius of curvature is denoted by $R, h$ is the length of the unbent arc, $h^{\prime}$ is the length of the bent arc, and $\xi$ is the horizontal displacement of the bottom of the arc. From Fig. 13, we see that the change in arc length is

$$
\delta h=h^{\prime}-h \approx \xi^{2} / 2 h
$$

so that the work, $W_{m}$, done in stretching the external magnetic field is

$$
\begin{gathered}
W_{m}=\text { stretching force } \times \text { distance stretched } \\
=\frac{B_{\text {ext }}^{2}}{2 \mu_{0}} \pi r^{2} \frac{\xi^{2}}{2 h},
\end{gathered}
$$

where $r$ is the mean arc radius. 
The centrifugal force density due to the curvature of the arc is $\rho U^{2} / R$. From Fig. 13, we see that $(R-\xi)^{2}+h^{2}=R^{2}$, giving

$$
\frac{1}{R} \approx 2 \frac{\xi}{h^{2}}
$$

so that the centrifugal force density, $F_{c}$, is

$$
F_{c}=2 \rho U^{2} \frac{\xi}{h^{2}} \text {. }
$$

The work per unit volume done by the centrifugal force is $\sim \int F_{c} d \xi$; however, we must multiply by a numerical factor $\sim 1 / 2$ to take into account the fact that the displacement of the arc is $\xi$ only at the bottom and decreases to zero on going to the top. Hence, we obtain $W_{c}$, the total work done by the centrifugal force, to be

$$
W_{c} \approx \frac{\rho U^{2} \xi^{2} \pi r^{2}}{2 h} .
$$

For stability, we need the work required to stretch the magnetic field to exceed the work done by the centrifugal force, i.e., $W_{m}>W_{c}$, or

$$
\frac{B_{\text {ext }}^{2}}{\rho \mu_{0}}>2 U^{2}
$$

which leads to (4).

\section{SPECUlation on the CaUse of Helical ROTATION OF ARC AT High MAGNETIC FIELDS}

A requirement for the axial magnetic field to stabilize the arc against kink or fire-hose instabilities is that the axial magnetic field be frozen into the arc. Dattner [25] described kink and sausage instabilities induced in a falling mercury jet where because of the long time scales the axial magnetic field was definitely not frozen into the mercury. In this case, as Dattner pointed out, when the arc develops a curvature, the upper part of the arc (which has conventional $J_{r}$ pointing in) has an azimuthal force $J_{r} B_{z}$ in the opposite direction of the azimuthal force acting on the bottom of the arc (which has conventional $J_{r}$ pointing out). The time scales in our experiment are much faster than in Dattner's mercury experiment, and the assumption that the magnetic field is frozen into our arc is clearly reasonable, since otherwise the magnetic stabilization scheme would not have worked.

However, presumably the magnetic field is not perfectly frozen into our arc and so to the extent that the axial magnetic field is imperfectly frozen there will be a twisting force on the arc. This torque will tend to make the arc helical and will also drive torsional Alfvén waves. The magnetic field evolution is determined by the induction equation

$$
\frac{\partial \vec{B}}{\partial t}=\nabla \times(\vec{U} \times \vec{B})-\nabla \times \frac{\eta}{\mu_{0}} \nabla \times \vec{B} .
$$

The magnetic Reynolds number, $\mathrm{R}_{M} \sim U L \mu_{0} / \eta$, which is the ratio of the convective RHS term to the diffusive RHS term, gives the extent to which the field is frozen into the plasma. Neither $U$ nor $\eta$ has been measured in this plasma, so only a rough estimate will be given. Assuming that the plasma density is $n \sim 10^{22} \mathrm{~m}^{-3}$, the atomic mass number is $\sim 30$, and using the cathode magnetic field $B \sim 0.08 \mathrm{~T}$, cathode radius of $3 \mathrm{~mm}$, and arc current of $1.2 \mathrm{kA}$ gives $U \sim V_{A} \sim 3 \times 10^{3}$ $\mathrm{m} / \mathrm{s}$. Using a typical arc resistivity of $\eta \sim 10^{-4} \Omega-\mathrm{m}$, and a typical scale length of $1 \mathrm{~cm}$ gives $\mathrm{R}_{M} \sim 0.3$. This must be considered a very rough estimate because of the uncertainties in $n, U, L$, and $\eta$, but clearly $\mathrm{R}_{M}$ is not likely to be larger than of order unity for the arc considered here (however, $\mathrm{R}_{M}$ will be larger for higher current arcs since $V \sim I, L \sim I$ ). Hence, as suggested above the stabilizing field is only partially frozen into the plasma. It is in fact surprising that the stabilization worked as well as it did here considering the relatively low value of $\mathrm{R}_{M}$ in this experiment.

\section{Discussion of BADISCHE STAHLWERKE ARC STABILIZATION EXPERIMENT}

A large scale arc control experiment somewhat related in concept to the work presented here was attempted-unsuccessfully - in 1980 by Kasper [30] at the Badische Stahlwerke in Kehl, Germany. The intended method, based on a patent by Stenkvist [31], was to control the arcs in an 80 ton, three-phase ac, 40-70 kA furnace in such a way that the arcs would not hit the wall and damage the refractory wall lining material. This experiment was unsuccessful in that no effect on the arc was observed. Using the stabilization concept presented here, it is possible to see in retrospect why the Badische Stahlwerke experiment (BSE) did not work.

Stenkvist's theory and the BSE assumed that the force causing a given arc to strike the wall was the $\vec{J} \times \vec{B}$ force arising from the interaction between the current flowing in the given arc and the magnetic field produced by the other two arcs of the three-phase furnace. Stenkvist's theory predicted that the radial component of a dc magnetic field produced by a coaxial coil underneath the furnace would counteract this force and instead cause the arcs to rotate in a circular trajectory away from the wall. For the 40-70 kA currents and $1.2 \mathrm{~m}$ arc-arc separation in the BSE, the field on a given arc due to its neighbor arcs was estimated [30] to be $10-20 \mathrm{mT}$. Thus a $2.6 \mathrm{~m}$ diameter coaxial dc electromagnet capable of producing a mainly radial field of $20 \mathrm{mT}$ at the arc was installed beneath the furnace (about $2 \mathrm{~m}$ below the arcs).

We have shown here that the force due to interaction with the rather distant neighbor arcs simply acts to instigate the kink and fire-hose instabilities and that to prevent these instabilities an axial stabilizing magnetic field of the order of the arc selfmagnetic field is required. This is essentially a factor of 10-20 greater than the BSE fields and also points in a different direction. For example, for a $40 \mathrm{kA}$ arc, the cathode spot radius will be $\approx 1.7 \mathrm{~cm}$, and if we assume that the average arc radius is double the cathode spot radius, we obtain an arc self magnetic field of $240 \mathrm{mT}$, an order of magnitude larger than the fields used in the BSE. Thus, the BSE magnetic field, intended as a redeflection system, was overwhelmed by the kink and fire-hose instabilities which can only be quenched by the much stronger axial field discussed here. The axial field does not redeflect the arc; rather it prevents it from bending in the first place. 


\section{SUMMARY AND CONCLUSIONS}

Application of an external magnetic field parallel to the arc current has been shown to stabilize a small atmospheric pressure high-current arc. In agreement with theoretical predictions, the required field is of the order of the self field of the arc. This experiment is small scale and so it is somewht speculative to presume that these results could apply directly to much larger industrial electric furnaces. To allow proper scaling an intermediate size experiment with a longer arc and at least $10 \mathrm{kA}$ should be performed (such an experiment is now being designed).

The mechanism of the fire-hose instability in the highcurrent arc has been presented showing that it is of comparable importance to the kink instability. The role of asymmetric return currents initiating the instability has been discussed. A high-frequency, stable helical rotation has been observed in a magnetized arc when the external magnetic field is relatively large.

\section{ACKNOWLEDGMENT}

The authors would like to thank B. Bangerter for his assistance in constructing the pulsed arc, F. Cosso for assistance with the pulsed electronics, M. Chamness for assistance with construction of the low-resolution video camera, $T$. Ochs of the U.S. Bureau of Mines for lending fiber optic isolation transceivers used for monitoring arc parameters, J. Gregoire of the NASA Jet Propulsion Laboratory for lending the high-speed video camera and crew, and R. Kasper for providing very helpful information on the Badische Stahlwerke experiment.

\section{REFERENCES}

[1] The Electric Arc Furnace-1990, Committee on Technology, Brussels, International Iron and Steel Institute, 1990.

[2] S-E. Stenkvist, "Single electrode d-c arc furnace,"Iron and Steel Engineer Magazine, vol. 62, pp. 50-54, 1985.

[3] T. L. Ochs, "Diagnosis of electrical noise in electric arc furnace operations," in Proc. 1987 IEEE Int. Conf. Plasma Science, p. 47.

[4] D. H. McQueen, "Noise from electric arc furnaces," Scand. J. Metallurgy, vol. 7, pp. 5-10, 1978.

[5] D. C. Strachan, "High-current, steel-cathode, free-burning arcs," J. Phys. D., vol. 10 , pp. $361-370,1977$.

[6] H. Maecker, "Plasmastromungen in Lichtbogen," Z. Physik, vol. 141, pp. $198-216,1955$.

[7] B. Bowman, G. R. Jordan, and F. Fitzgerald, "The physics of highcurrent arcs," J. Iron Steel Inst., pp. 798-805, June 1969.

[8] B. Bowman, "Measurements of plasma velocity distributions in freeburning DC arcs up to 2160 A," J. Phys. $D$., vol. 5, pp. 1422-1432, 1972.

[9] G. R. Jordan, B. Bowman, and D. Wakelam, "Electrical and photographic measurements of high-power arcs," J. Phys. D., vol. 3, pp. 1089-1099, 1969.

[10] H. Edels, "Properties of the high pressure ultra high current arc," in Proc. 11th Conf. Phenomena in Ionized Gases (Prague), 1973, vol. 2, pp. 9-59.

[11] M. Irie and M. R. Barrault, "Flow velocity measurement in high-current arcs using Doppler-shifted plasma scattering," J. Phys. D., vol. 10, pp. 1599-1605, 1977.

[12] J. W. McKelliget and J. Szekely, "A mathematical model of the cathode region of a high intensity carbon arc," J. Phys. D., vol. 16, pp. 1007-1022, 1983.

[13] G. R. Jones, High Pressure Arcs in Industrial Devices. Cambridge: Cambridge Univ. Press, 1988.

[14] T. L. Ochs, A. D. Hartman, and S. L. Witkowski, "Waveform analysis of electric furnace arcs as a diagnostic tool," U.S. Bureau of Mines Report of Investigations no. 9029, 1986.

[15] Ref. [13], pp. $176 \mathrm{ff}$.

[16] T. S. Mel'nikova, "Instabilities of an electric arc," High Temperature, vol. 18 , pp. $720-725,1980$.

[17] B. P. Peregud and S. V. Ponedilko, "MHD instability in a stream of metal in arc smelting," Sov. Phys. Tech. Phys., vol. 25, pp. 1230-1234, 1980.

[18] R. W. Montgomery and C. M. H. Sharp, Brit. J. Appl. Phys., vol. 2, pp. 1345-1348, 1969.

[19] For a standard text-book derivation of the kink instability, see G. Schmidt, Physics of High Temperature Plasmas, 2nd ed. New York, Academic Press, 1979, chap. V.

[20] ibid., p. 258.

[21] R. C. Davidson and H. J. Volk, "Macroscopic quasilinear theory of the garden-hose instability," Phys. Fluids, vol. 11, pp. 2259-2264, 1968.

[22] P. M. Bellan, "Method and apparatus for stabilizing electric furnace arcs using an externally applied magnetic field," U.S. patent $4815097,1989$.

[23] G. A. Sawyer, P. L. Scott, and T. F. Stratton, "Experimental demonstration of hydromagnetic waves in an ionized gas," Phys. Fluids, vol. 2, pp. 47-5l, 1959.

[24] H. Yoshikawa and M. Irie, "Characteristic voltage fluctuations in low pressure high current arcs," in Proc. XVII Int. Conf. Phenomena in Ionized Gases (Swansea, U.K.), 1987, part I.

[25] A. Dattner, "Current-induced instabilities of a mercury jet," Arkiv for Fysik, vol. 21, pp. 71-80, 1961.

[26] J. Spencer, J. Parry, G. R. Jones, "Complex aspects of arc behavior in a rotary arc circuit breaker," in Proc. 8th Int. Conf. Gas Discharges and their Applications, 1985 , p. 133.

[27] G. R. Jones, "High current arcs at high pressures," in Proc. XVI Intl. Conf. Ionized Gases (Dusseldorf), 1983, invited paper.

[28] G. R. Jones, C. Eng, J. L. Leclerc, and M. R. Smith, "Self-magnetic effects in a model gas-blast circuit breaker at very high currents," Proc. Inst. Elec. Eng., vol. 129, pp. 611-618, 1982.

[29] G. R. Jones, G. G. Shishkin, and S. Taylor, "Oscillations and correlation analysis fluctuations in high current, convection controlled arcs," in Proc. XVIII Intl. Conf. Phenomena in Ionized Gases (Swansea, U.K.), 1987.

[30] R. Kasper, "Elektromagnetische Beeinsflussung des Lichtbogens in Elektroofen" (trans: "Electromagnetic control of arc flare in electric furnace,"), Bundesministrium fur Forschung und Technologie, Bonn, Germany, Technical Report BMFT-FB-T83-282, 1983

[31] S.-E. Stenkvist, U.S. patent 4034 146, 1976

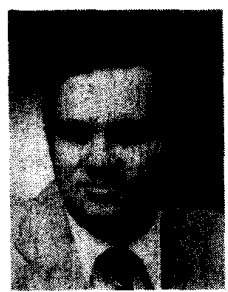

Paul M. Bellan was born in Winnipeg, Manitoba, Canada, in 1948. He obtained a B.Sc.(Hon.) degree from the University of Manitoba in 1970 and the M.A. and Ph.D. degrees in plasma physics from Princeton Unversity in 1972 and 1975.

After holding a postdoctoral position at the Princeton Plasma Physics Laboratory, he joined the Caltech faculty in 1977, where he is now a Professor of Applied Physics. His research activities have included experimental and theoretical studies of lower hybrid wave propagation in plasmas, driftAlfvén waves, laser-induced fluorescence of plasmas, stochastic ion heating by coherent drift waves, magnetic helicity injection into tokamaks, spheromak injection into tokamaks, RF current drive in tokamaks, and the stability of arcs. Dr. Bellan is a fellow of the American Physical Society.

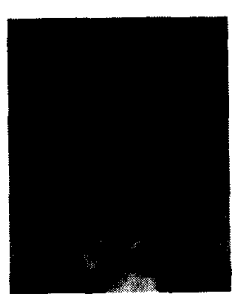

Jay W. Higley was born in St. George, UT, on February 16, 1969. He received the B.S. degree in applied physics from the California Institute of Technology, Pasadena, in June 1991.

Currently he is working as a Junior Analyst with XonTech, Inc., Los Angeles, CA 